pneumoniae. Practices did not differ significantly among the different groups of paediatricians.

Conclusion Standardized management of parapneumonic effusion, including routine thoracentesis and more consistent prescription of antibiotics, is needed.

\section{PO-0234 ADOPTED CHILDREN AND TINEA CAPITIS: SYSTEMATIC SCREENING NEEDED}

D Dupont, F Peyron, S Picot, M Wallon, AL Bienvenu. Parasitology Mycology, Hopital de La Croix Rousse, Lyon, France

\subsection{6/archdischild-2014-307384.885}

Background/aims Dermatophytes are keratinophilic fungi responsible for skin, nail and scalp infections. Over the past few years, a rising incidence of dermatophytoses has been reported, particularly due to infected migrants coming from developing countries. In this study, we studied dermatophytes' prevalence in a cohort of international adopted children.

Methods This retrospective study has been carried out in the Parasitology - Mycology clinic of La Croix Rousse University Hospital in Lyon, France from 1998 to 2012. Biological samples of scalp or hair have been analysed according to standard mycological procedures.

Results In total 101 children coming from Africa, and the Caribbean were sampled. 44 children had dermatophytosis (44/101, 43.6\%) eg Trichophyton soudanense, Trichophyton violaceum, Trichopyton tonsurans, Trichophyton rubrum and Microsporum audouinii. The clinical presentation of these patients was variable, including squamous scalp lesions without alopecia $(n=20)$, scalp alopecia-causing lesions $(n=6)$, squamous lesions scalp associated with tinea corporis $(n=5)$, tinea corporis $(n=4)$ and crusty scalp lesions $(\mathrm{n}=1)$. In seven cases we reported completely asymptomatic patients $(15.9 \%, 7 / 44)$. Systematic examination of their families led to the discovery of nine cases $(20.5 \%, 9 / 44)$ of family contamination. The therapeutic success rate was close to $80 \%$ following first line of treatment.

Conclusion We demonstrated that dermatophytoses often have a silent clinical presentation and, in approximately $20 \%$ of cases, cause family contamination. This study highlights the importance of the clinical examination of children and families, as well as systematic sampling of children, to avoid dermatophyte transmission to other family members.

\section{PO-0235 DECREASE IN INVASIVE PNEUMOCOCCAL DISEASE (IPD) AFTER INTRODUCTION OF PCV13 IN AN AREA WITHOUT SYSTEMATIC VACCINATION}

${ }^{1} \mathrm{M}$ Fernandez de Sevilla, ${ }^{1} \mathrm{~J}$ Martinez Osorio, ${ }^{1} \mathrm{JJ}$ Garcia-Garcia, ${ }^{2} \mathrm{~F}$ Moraga, ${ }^{3} \mathrm{P}$ Ciruela, ${ }^{4} \mathrm{~A}$ Dominguez, ${ }^{5} \mathrm{~A}$ Díaz, ${ }^{6} \mathrm{C}$ Muñoz-Almagro. ${ }^{1}$ Pediatric, Hospital Sant Joan de Déu, Barcelona, Spain; ${ }^{2}$ Pediatric, Hospital Vall d'Hebron, Barcelona, Spain; ${ }^{3}$ Department of Health Generalitat de Catalunya, General Subdirectorate of Surveillance and Response to Emergencies in Public Health, Barcelona, Spain; ${ }^{4}$ Department of Health, Universitat de Barcelona, Barcelona, Spain; ${ }^{5}$ Pediatric, Hospital de Nens, Barcelona, Spain; ${ }^{6}$ Microbiology, Hospital Sant Joan de Déu, Barcelona, Spain

\subsection{6/archdischild-2014-307384.886}

Background and aims To analyse the clinical presentation and serotype distribution of IPD, before and after PCV13 introduction.
Methods Prospective study comprising all children S. pneumoniae in a sterile fluid sample. The vaccination coverage was estimated by a control sample paired by age, sex and risk of IPD.

Results We included 319 patients during 2007-2009 and 81 patients in postvaccine period. A decrease in IPD was observed (106 episodes/year in prevaccine versus 40 episodes/year in postvaccine period) mainly due to decline of serotype 19A (decrease of $78 \%$ ) and serotype 1 (decrease of 71\%). Serotype 3 decrease was also observed (39\%).

PCV13 serotypes remained stable (70\% vs $76.6 \%)$. The most frequent serotypes during prevaccine period were: 1(21\%), 19A $(16 \%)$ and $3(12 \%)$ and during postvaccine period: 3 (26.5\%), 1 (20\%), and 19A(12.5\%). There were no serotypes replacement.

Pneumonia was the main clinical presentation in both periods.

PCV13 coverage during postvaccine period was $48 \%$.

31/81 patients were vaccinated with PCV13. 7 cases were considered complete vaccine failure (5 due to serotype 3 ).

Conclusions An important global decrease in IPD was observed, mostly in those cases produced by serotypes 19A and 1. Pneumonia was the main clinical presentation. There was no serotypes replacement. Some cases of vaccine failure were observed.

\section{PO-0236 ANALYSIS OF ANTIBIOTIC USE IN ACUTE BRONCHIOLITIS}

${ }^{1} \mathrm{JC}$ Flores-Gonzalez, ${ }^{1} \mathrm{~B}$ Serrano-Moyano, ${ }^{1} \mathrm{JJ}$ Perez-Guerrero, ${ }^{1} \mathrm{E}$ Palma-Zambrana, ${ }^{1} \mathrm{FJ}$ Dávila-Corrales, ${ }^{1} \mathrm{~L}$ Garcia-Garcia, ${ }^{1} \mathrm{M}$ A Matamala-Morillo, ${ }^{1} \mathrm{P}$ Rodriguez-Campoy, ${ }^{1} \mathrm{RM}$ Garcia-Ortega, ${ }^{1} \mathrm{P}$ Comino-Vazquez, ${ }^{2} \mathrm{R}$ Bulo-Contreras, ${ }^{2} \mathrm{AM}$ Lechuga-Sancho. ${ }^{1}$ Pediatrics Department, Hospital Universitario Puerta Del Mar, Cádiz, Spain; ${ }^{2}$ Farmaceutic Department, Hospital Universitario Puerta Del Mar, Cádiz, Spain

\subsection{6/archdischild-2014-307384.887}

Background and aims To evaluate the use of antibiotics and its relation to the length of stay (LOS) in patients hospitalised with acute bronchiolitis (AB).

Patients and Method In a context of a randomised, controlled, double-blind clinical trial, 177 infants hospitalised with $\mathrm{AB}$ in two epidemics (2011-2013) were included. They were classified according to the scale of Wood- Downes modified by Ferres (WDF), in mild (MiB), moderate (MB) or severe (SB). The primary outcome was LOS. We evaluated the antibiotic used and CRP. Data were analysed with SPSS17.

Results There were 7.9\% MiB, 83.6\% $\mathrm{MB}$ and $8.5 \% \mathrm{SB}$. Of them, $21.4 \%$ of $\mathrm{MiB}, 14.2 \%$ of $\mathrm{MB}$ and $66.7 \%$ of the $\mathrm{SB}$ received antibiotic therapy. The most commonly used antibiotics were amoxicillin-clavulanate, cefotaxime and azithromycin. The most frequent reasons were clinical worsening measured by WDF scale, increased CRP and fever. The mean LOS for acute bronchiolitis without antibiotics was 4.51 days and with antibiotics 7.84 days $(p=0.001)$. The use of antibiotics in SB was significantly higher than in $\mathrm{MB}(\mathrm{p}=0.001)$. In the group of $\mathrm{MB}$, the mean LOS was 4.44 days (no antibiotics) vs 7.29 (antibiotics), $\mathrm{p}=0.001$. In the group of BG, the mean LOS was 9.4 days vs. 13.5 days, $\mathrm{p}=0.53$. There were statistically significant differences in elevated CRP and the use of antibiotics ( $\mathrm{p}=$ 0.005).

Conclusions $\mathrm{The} \mathrm{AB}$ who received antibiotics had significantly more LOS. There were statistically significant differences to received antibiotics in $\mathrm{MB}$, but not in $\mathrm{SB}$, perhaps because the LOS of this group is high. 